WwW.iibpas.com

\title{
MEDICAL IMAGE PROCESSING AND ANALYSIS USING DEEP LEARNING APPROACH
}

\section{ATISH PESHATTIWAR ${ }^{1^{*}}$, BUVANESWARI.B ${ }^{2}$, POORNIMA H. $\mathbf{N}^{3}$, NEETA BHUSAL SHARMA $^{4}$, SYED KHASIM ${ }^{5}$ AND PRAVEEN KUMAR ${ }^{6}$}

1: Assistant Professor in Electronics Engineering at Yeshwantrao Chavan College Of Engineering Nagpur Maharashtra, India

2: Professor in Information Technology at Panimalar Engineering college, Chennai, India

3: Research Scholar in Information Science at AMC College of Engineering, Bangalore, Karnataka, India

4: Assistant Professor in Computer Science \& Engineering at Shri Ramswaroop Memorial University Lucknow, India

5: Professor in Computer Science and Engineering at Dr.Samuel George Institute of Engineering \& Technology, Markapur, Prakasam Dt, Andhra Pradesh, India

6: Assistant Professor in Electrical Engineering at BIT Sindri, Dhanbad, Jharkhand, India

*Corresponding Author: Atish Peshattiwar; E Mail: atishp32@gmail.com

Received 20 ${ }^{\text {th }}$ July 2021; Revised 22 ${ }^{\text {nd }}$ Aug. 2021; Accepted $30^{\text {th }}$ Sept. 2021; Available online $1^{\text {st }}$ Nov. 2021 https://doi.org/10.31032/IJBPAS/2021/10.11.1065

\begin{abstract}
The use of profound having to learn techniques within therapeutic imagery treatment but also assessment having had overall huge consequence. Imaging identification, imaging augmentation, including classifier but rather modeling are common procedures throughout therapeutic visual handling but instead interpretation using profound network techniques. This same absence of appropriate labeled experimental datasets has been commonly highlighted as significant difficulty towards supervising profound understanding. Humans seek help solve fundamental challenges to developing global massive neuronal architectures utilizing either relatively minimal volume available labelled material within these study. Using the profound dynamic learned approach, researchers provided a flexible architecture to healthcare picture cleaning \& evaluation.(1) Implementing software sophisticated energetic learners
\end{abstract}


technique could separate particular locations that attention (Locations information participation) out unprocessed diagnostic images with very least amount information tagged metadata feasible; (2) A discursive accusatorial connection can be used to improve the direct comparison, picture quality, but instead reflectivity of segmentation process Region of interest; (3) A Blistering speed Preparation Studying (Antigen) approach can be used to operate medicine svm classifier and perhaps statistical basic functions besides toolset throughout profound synaptic systems from pinnacle to base phase. Furthermore additional, Classification Dynamic Maps (CAM) being used furthermore this architecture that display each features need better comprehend that requirement on profound healthcare picture processing jobs and offer suggestions for clinical usage.Furthermore demonstrate this same efficacy underlying this same developed methodology, researchers implement it furthermore quantitative skeletal ageing appraisal (Complainant) problem with this same Begins by creating datasets as well as get best-in-class results. This suggested paradigm may successfully use towards healthcare imagery assessment tasks, according upon practical outcomes.

\section{Keywords: Biomedical imaging preprocessing; Dense multilayer systems; Pacing direction} optimization

\section{INTRODUCTION}

However though bigger unsupervised sample volumes become generally readily accessed, and paucity adequate labelled research samples has been frequently noted and fundamental difficulty whenever using profound learned algorithms toward healthcare imagery processing. Medicinal picture labelling by hand involves costly, moment, therefore needs skilled clinicians [1-3]. So result conclusion, particularly profound therapeutic visual computing assignments, decreasing this same quantity of labelled knowledge becomes critical, because forming such neuronal structure without little labelled information remains difficult. Throughout broad sense, photograph categorization, illustration improvements, as well as prognostication are used throughout medicine photograph characterization but rather degeneration using depths having to learn techniques, these rather as cartilage young and middle evaluations, bronchiolitis diagnosis on shoulder Cin, histologic large scale designation, prediabetes proliferative diabetic characterization, cerebral lesion evaluation, and so through [4]. With its anomaly of [5], that further performs three studies of varying preparation but rather interpretation responsibilities throughout separate configurations but rather systematizes this same procedure of transaction studying, those current writings 
through this same profession of clinical photograph handling but also analyzation have been concentrated on yet another characteristic these as segmentation, detection, and classification [6]. Humans expand their approach underlying previous study that turn that as well become comprehensive foundation towards medicinal imagery assessment.

Retrieving particular Profit margin using unprocessed hospital picture might save considerable lot of time as well as effort when it comes to scanning. Furthermore, removing unnecessary contamination from diagnostic images might help enhance modelling performances during this same projection process [7]. Regardless overall diversity between intensity, cleanliness, the intensity among healthcare pictures, that becomes difficult can build effective softback layer between unprocessed healthcare pictures towards particular Regions of interest [8]. Despite the fact that depths picture separation activities yield impressive results, very significant volume highly annotation photos is required. Branding effort involves relatively demanding throughout actuality as well as sometimes have specialist information [9-11]. Considering similar setting, researchers present another profound passive tracking technique which reduce labeling load whilst ensuring prototype correctness with that little labelled information as feasible throughout attempt effectively separate particular N'est pas encore form unprocessed hospital pictures. This seems vital must describe basic foundational functionality underlying profound neuron systems, where provides equal greatest up-to-date classifications structure but also would being translated towards area with healthcare photographs as well as goal, providing much deeper comprehension how picture segments technique. Across numerous sizable photo classification problems, massively interconnected multilayer neurons having demonstrated impressive precision with spectacular resolution characteristics. Similarly, when restore substantially comprehensive material through repeating properties files, jump connectors connecting this same downwards sweeping through this same back testing network have been commonly used.

With take use utilizing subsurface DenseNet's strong capabilities that swiftly as well as completely attack problematic objects, this same transitioning upward as well as movement downstream frames suggested previously [12] being used with entirely integrated. Thoroughly attached 
layers (Newcastle united) that conduct picture conceptual classification.

\section{METHODOLOGY}

This same following was this same journal's key commitments: Researchers present another new AL technique as well as employ intelligence toward the job that conceptual identification in hospital photographs. Researchers would considerably reduce human annotated effort despite ensuring network correctness besides combining depths Cetera plus this same Queries Following Review panel (Adorned) method. Towards improve picture resolution as well as correct increasing demands for greater safety medicinal imaging, human's present offer a special unique medicinal imaging allow appropriate system mainly comprises essentially using Graphene. Humans present innovative Pragmatic technique enabling fine-tuning off-the-shelf hidden Units för particular applications thus ensuring that models achieves remarkable results when relative on traditional shallow transferable acquisition methods (Figure 1).

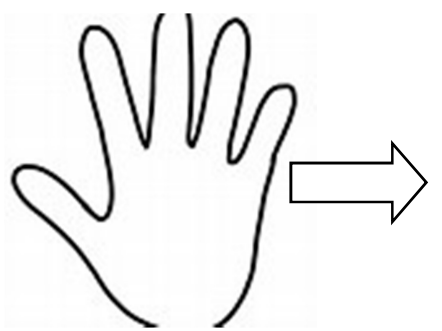

Another basic postulate of this same Cetera design suggests how this same learning may select certain facts during lexical interpretation because carry computer highest plentiful content. Many picture segments algorithms based around Alc methodologies having subsequently become presented. Actively educators pick unmarked facts that include significant material should become labelled through divination

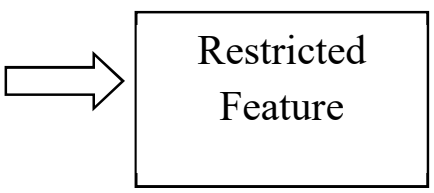

Figure 1: Proposed architecture

throughout overall great proportion most situations using probability sampled techniques. Another main goal underlying a probability picking method simply quantify dataset volatility. Present systems frequently employ ideas such include minimum content, data diversification, and quantization towards solve these issue. Sensitivity, transversal free energy, and so on. Their goal, the other hand, simply generate choose photos using the 
Straightforward approach, which would employ rather numerous single paradigm. Everyone commission membership under especially single being schooled using this same comparable material. Second inquiry being picked using this same maximum difference approach. Thus within field for picture feature extraction analysis. Under overall Cetera system, there will important should intelligently choose samples during their retraining step. When all of this comes down selecting this same highest meaningful request instances about whatever entire commission differs about maximum, humans employ probability selection. Throughout every picture separation challenge, researchers must determine this same database's confidence, or overall amount significant difference. Because researchers educated multiple group smart people within standing committees, everyone person acquires this same increased features about this same initial photograph, clearly illustrated with Example 2.

\section{RESULTS AND DISCUSSIONS}

Employ their system towards diagnosis job generating Bba utilizing using publicly published information through this same removes certain guesswork Skeletal Aging Challenges that demonstrate its efficacy effective productivity.
Mitochondrial Maturity was screening typical healthcare technique when determining their toddler's bony maturity degree. Mismatch among both academic generation as well as this same formation for bony maturity suggests orthopedic problems. Oncologists might assess this development problem, keep track of prescription testosterone treatment, and thus forth. Bba can also be used to determine adulthood stature. When conduct BAA leveraging my system, they must partition palm return through investment using unprocessed Anti pictures, improve palm Return of investment accuracy, effectively estimate skeletal ageing employing transferable learned. Throughout development collection, there are 12611 instances throughout those palm rays supplied through Representatives. As illustrated in Figures 2, total skeleton maturity ranged 227 decades.

For proactively divide hands Return on investment form uncompressed Anti pictures, researchers deploy Local club both effective manual radiographic segmented networks using the Fuzzy logic control approach. Protocol shows that retraining technique with further depth. Throughout my tests, you used a three-person team. Trained Club name using its framework shown throughout Figure 2, plus they use various 
arbitrary seeding for establishes learning system variables all that therefore individual characteristic matrices remain distinct. This same hypothesized deeper Cetera system picks this same highest meaningful information from any unorganized sample from each loop of that algorithm then requests another person spokesperson additional handwritten masking.

Researchers physically comment 100+ basic Anti - anti pictures during an instructional initial part. With every learning repetition, simulations software should inform readers whether information remain critical during trainee, after whom humans provide actual bottom truths about actual Anti - anti pictures as well as submit those towards labelled test set. Researchers employ those learned DenseNets with deduce incoming training examples as well as manually evaluate overall segmented findings so see if network adequately resolved. Researchers noticed how computer classification outputs for such unprocessed pictures containing even those highest distinct characteristics were distinct. Such occurrence indicates that its suggested Cetera structure plus Fuzzy logic control approach seems efficient. Figure 3 depicts overall outcomes of classification. Humans clipped individual finger Regions of interest form overall whole picture while segregating hands stencils, notably indicated with this same final lines. Net for construct robust thumb division networks using just 200 tagged palms. Return on investment but also other AL method. Overall classification data have been demonstrated throughout this same right edge. This same effectiveness of separation will clearly enhanced by using overall Cetera retraining technique. These suggests also that suggested Cetera method may successfully pick information during segmented development set as well as create higher segmented outputs.

Researchers quite well this same settings inside every phase simultaneously fixing relevant characteristics exterior within that blocked that show that suggested Speed Ordering Learned technique surpasses traditional transferred learned approaches. Every transferring learned system were developed on improved finger radiographic scaled down 298296 pixels. When comparison against alternative transferable learned scenarios, my system features absolute greatest results within every section from given database, including entire, adult, as well as woman generations. Another finding shows this despite while researchers fine-tuned considerably higher number learning features across an entire Cns with 
this same start, i.e., starting from this same entering brick through so well subsequent stages, modelling degradation would significantly reduce. This implies that really well many levels towards early start of each process may compromise really well settings. So well very limited range more characteristics, one this same other hand, typically enough enabling modeling retraining. Considering that being potentially significant disparity across natural versus professional pictures, similar situation may arise with transferred learned upon healthcare photo processor applications. Figure 4 compares on algorithms using various transferred learned techniques.

Using Speed Sequence Filtering may significantly enhance modelling effectiveness while lowering MAE. By looking through this same second 3 points throughout Figures 5 and 6 humans can see whether with nation classifier performs significantly when we use improved palm categorization pictures. Across general, masculine, nor feminine generations, this same suggested Bba framework had a 7.664, 5.991, and 6.263 days, accordingly. Switching people between men's and women groups, furthermore, may enhance modeling effectiveness marginally. Another rationale for inequality seems because throughout medical practice, boys and girls groups usually assessed using distinct benchmarks.

Throughout terms overall Madam Assessment as well as coaching duration, this then appears apparent why their suggested methodology outperforms previous techniques. Humans concluded as this same presented $\mathrm{Bba}$ to poisomerase strategy considerably adapts investment is high features toward diagnostic images processing challenges as well as speeds this same instructional operation especially comparable using several prior $\mathrm{Ba}$ approaches using transferred learned within [13]. Algorithm suggested methodology effectively avoids regional least traps as well as causes this same network should merge across multiple workouts. Furthermore, my network proven useful everything statistics submitted inside this database thus obtained substantially elevated results when comparison to this same other approaches that were developed on such arelatively short sample using [14].

Humans relate these approaches against that leadership chart provided somewhat online Begins by creating task webpage that properly highlight evaluating performance using that suggested strategy towards BAA. Figure 7 shows that results are ranked 4th and 9th in male and feminine generations, respectively. Underlined Regions throughout 
indicate critical sections from these picture to doing overall bones aged calculation. Regarding the human skeletons, Camera pays lesser consideration toward forearm than towards metacarpal vertebrae, suggesting therefore carpus vertebrae seem more significant when determining skeletal maturity under their approach. Cfm treats a broad array the finger fractures adult women, as well as the tails of distal phalanx, metatarsals joints, but also metacarpals. Lateral ligaments, with the other hand.

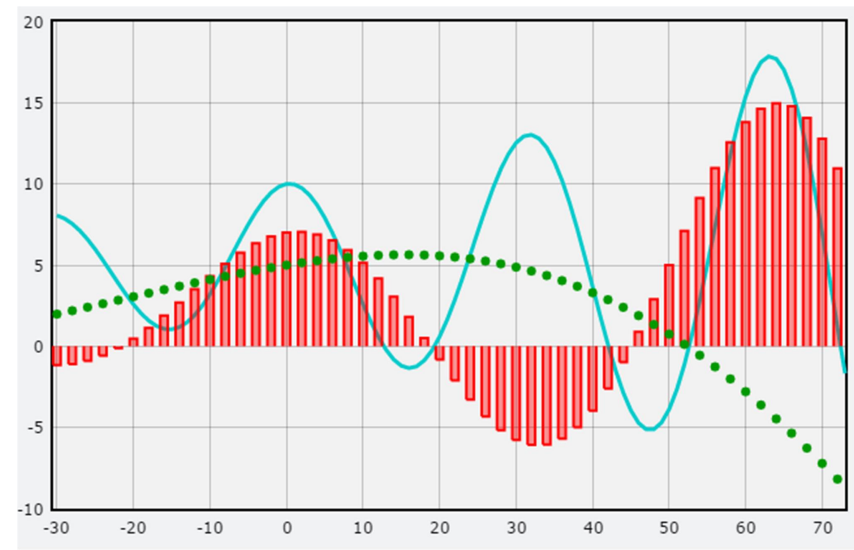

Figure 2 Skeleton maturity ranges
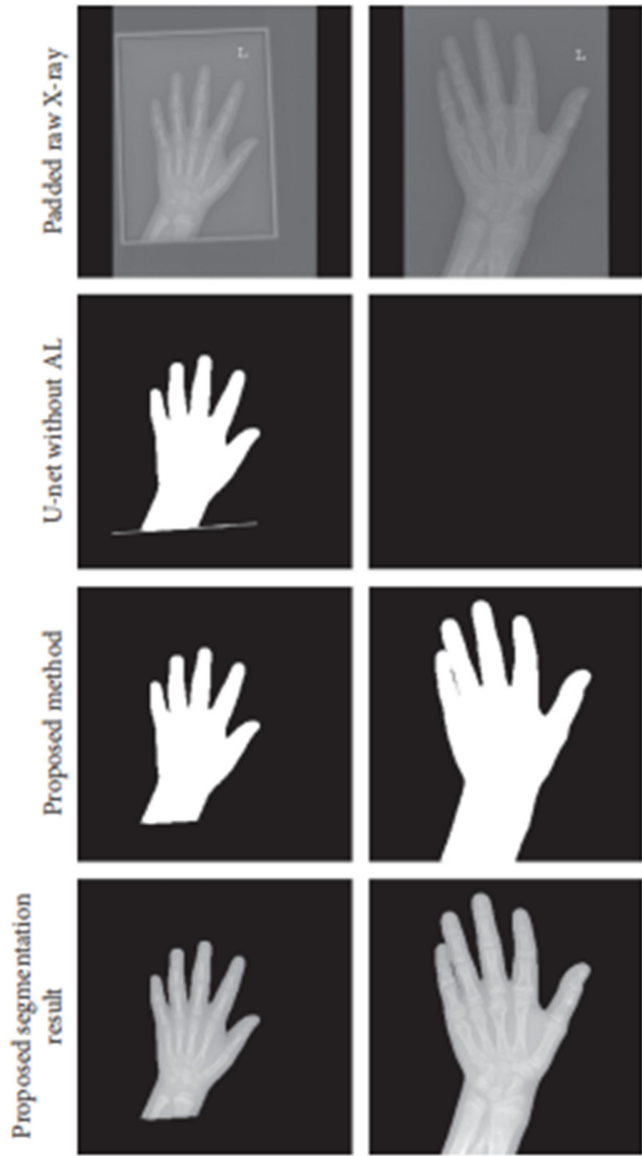
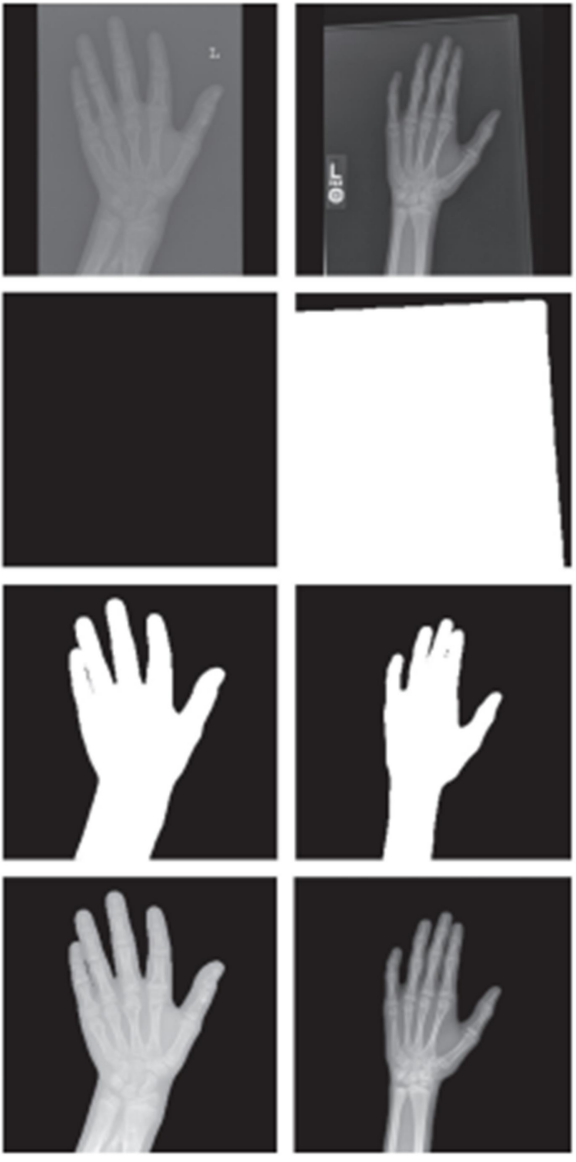

Figure 3: Overall outcomes of classification
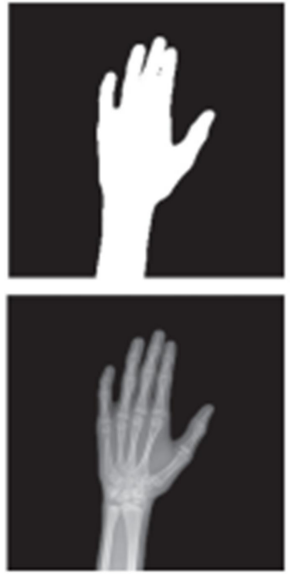
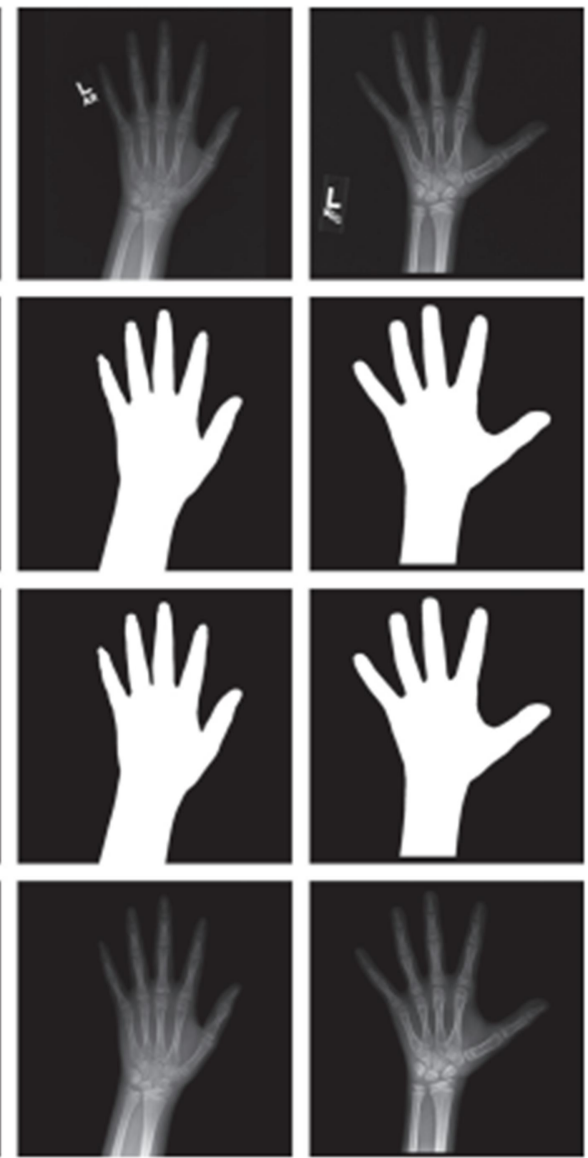


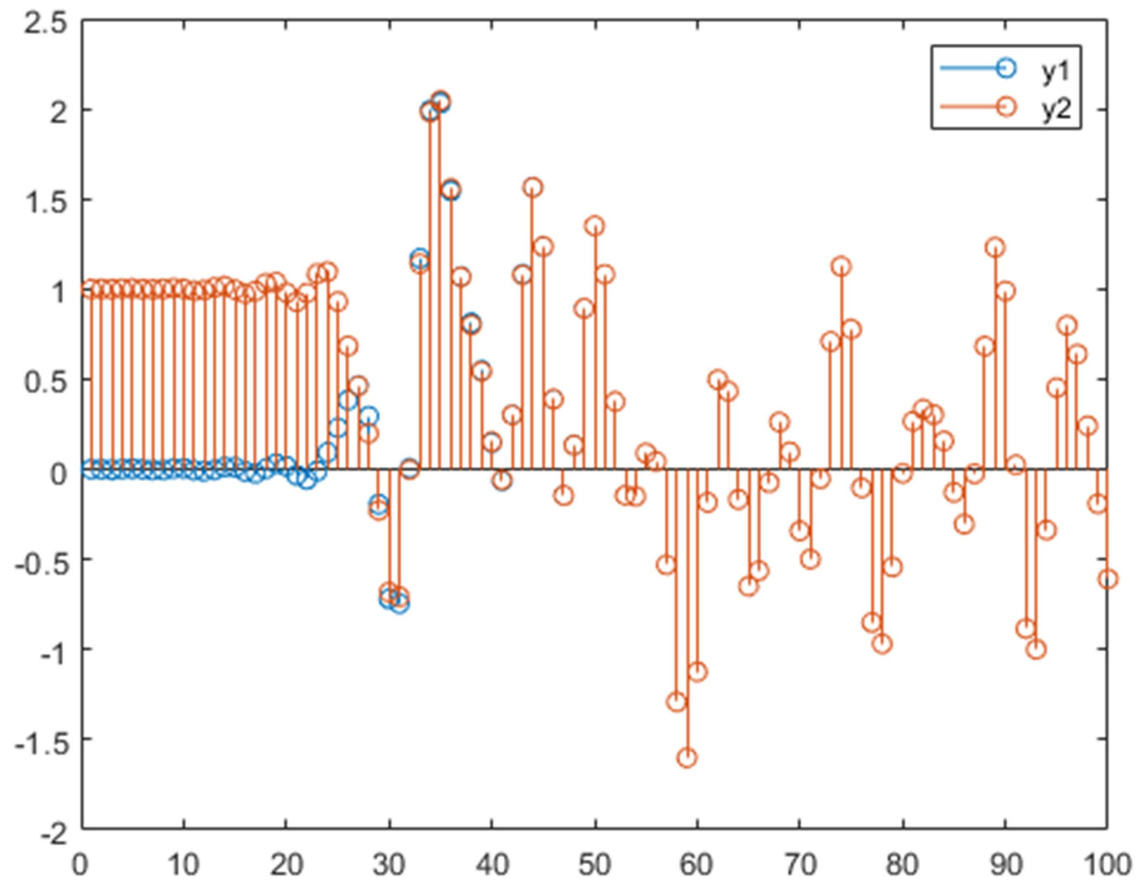

Figure 4: Compares on algorithms using various transferred learned techniques

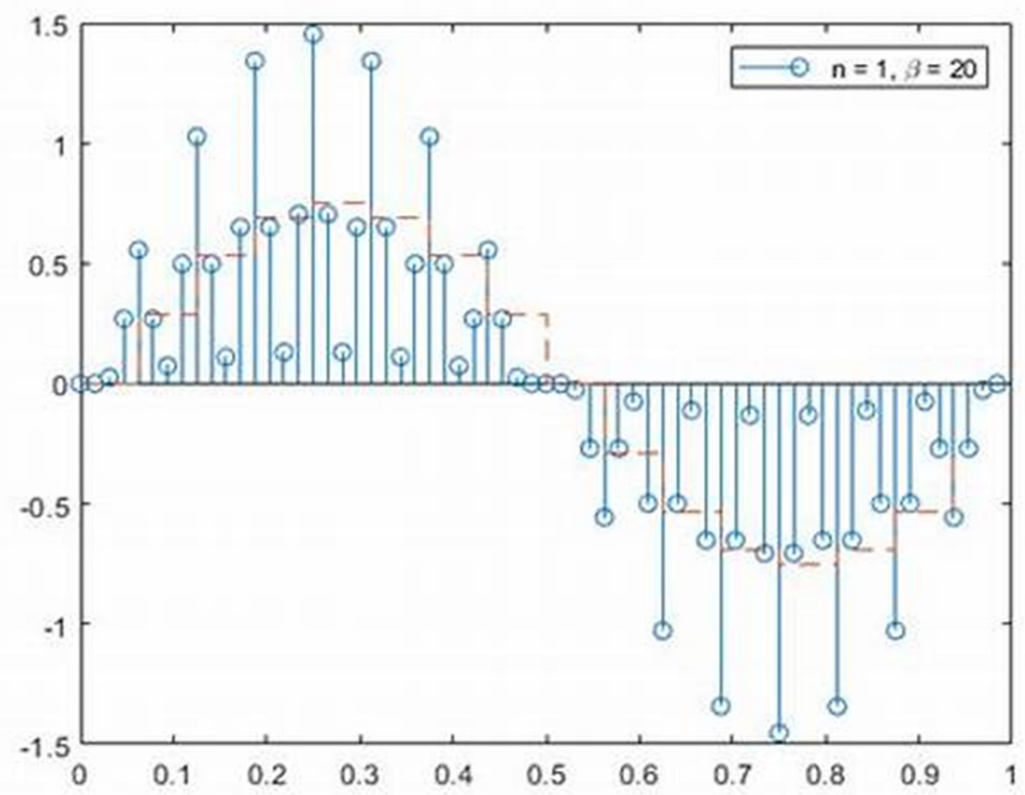

Figure 5: Speed Sequence Filtering 


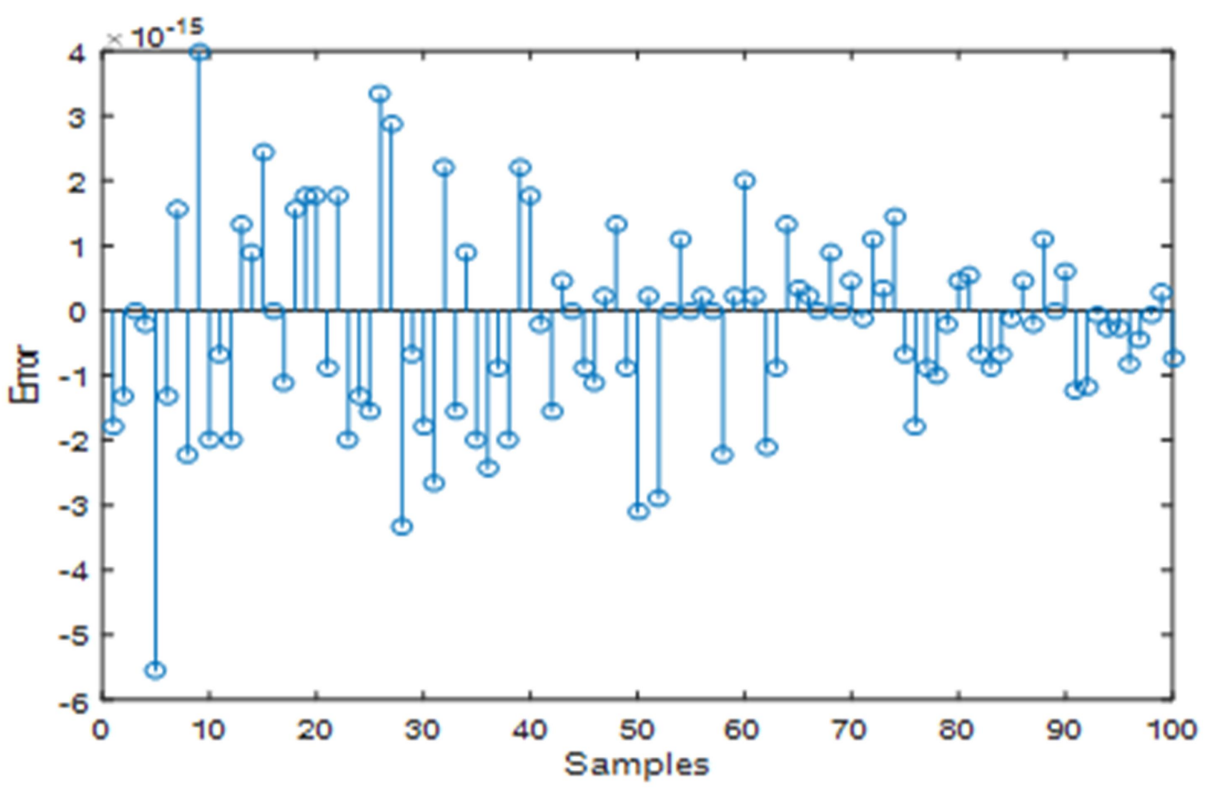

Figure 6: Speed Sequence Filtering based on errors

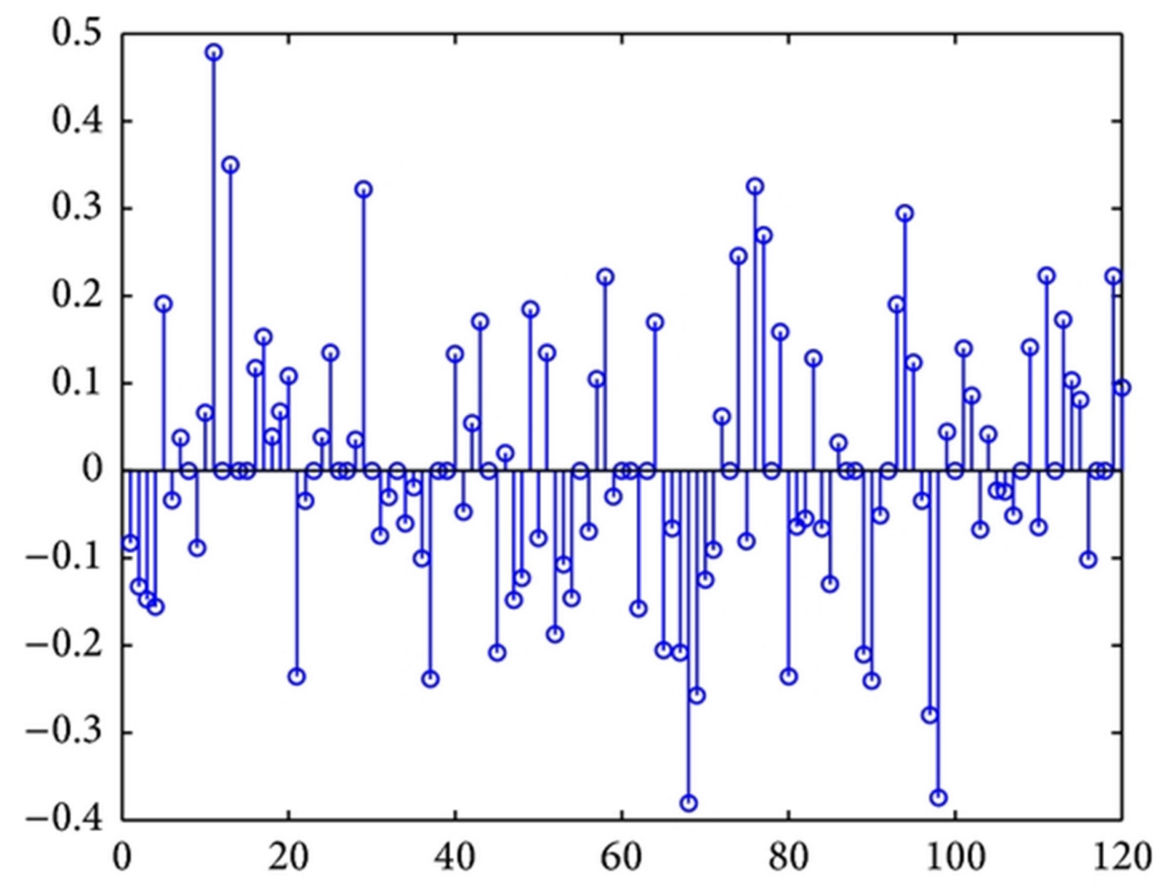

Figure 7: Overall bones aged calculation

CONCLUSION

Designers developed more flexible architecture towards health picture cleaning but also interpretation leveraging profound learned techniques in current study. Human's present learning profound Cetera methodology based information preparation that proactively selects this same highest meaningful material via annotations as well as segmentation tailored Revenue employing 
FCDenseNet. Nvidia technique also used help improve the appearance of clinical images. Researchers suggest using Democrat could perfect that off Network as well as execute prediction using such healthcare picture analysis either classifying job. Researchers also use Video feed to display deep Network as well as illustrate whether parts from picture remain important enabling browser systems to do particular healthcare data analysis but instead image processing. Researchers apply algorithm using given Cana challenge using this same RSNA collection that demonstrate overall efficacy overall reliability using suggested architecture. Using men as well as women populations, their approach obtains an I'r of 5.991 as well as 6.263 weeks, respectively, which is equivalent with province efficiency across high big corpus. That shall keep, researchers think, may be used for additional healthcare face recognition problems.

\section{REFERENCES}

[1] Jifara W, Jiang F, Rho S, Cheng M, Liu S. Medical image denoising using convolutional neural network: a residual learning approach. The Journal of Supercomputing. 2019 Feb; 075(2): 704-18.

[2] Chen JI. Design of Accurate Classification of COVID-19 Disease in X-Ray Images Using Deep Learning Approach. Journal of ISMAC. 2021 Jun 15; 3(02): 132-48.

[3] Vijayalakshmi A. Deep learning approach to detect malaria from microscopic images. Multimedia Tools and Applications. 2020 Jun; 79(21): 15297-317.

[4] Maier A, Syben C, Lasser T, Riess C. A gentle introduction to deep learning in medical image processing. ZeitschriftfürMedizinischePhysik. 2019 May 1; 29(2): 86-101.

[5] Wang J, Zhu H, Wang SH, Zhang YD. A review of deep learning on medical image analysis. Mobile Networks and Applications. 2021 Feb; 26(1): 351-80.

[6] Zhou T, Ruan S, Canu S. A review: Deep learning for medical image segmentation using multi-modality fusion. Array. 2019 Sep 1; 3: 100004.

[7] Awais M, Müller H, Tang TB, Meriaudeau F. Classification of sdoct images using a deep learning approach. In2017 IEEE International Conference on Signal and Image Processing Applications (ICSIPA) 2017 Sep 12 (pp. 489-492). IEEE.

[8] Razzak MI, Naz S, Zaib A. Deep learning for medical image 
processing: Overview, challenges and the future. Classification in Bio. Apps. 2018: 323-50.

[9] Latchoumi, T. P., Reddy, M. S., \& Balamurugan, K. (2020). Applied Machine Learning Predictive Analytics to SQL Injection Attack Detection and Prevention. European Journal of Molecular \& Clinical Medicine, 7(02), 2020

[10] Bhattacharya S, Maddikunta PK, Pham QV, Gadekallu TR, Chowdhary CL, Alazab M, Piran MJ. Deep learning and medical image processing for coronavirus (COVID-19) pandemic: A survey. Sustainable cities and society. 2021 Feb 1; 65: 102589.

[11] Garikapati, P., Balamurugan, K., Latchoumi, T. P., \&Malkapuram, R. (2021). A Cluster-Profile Comparative Study on Machining AlSi $7 / 63 \%$ of $\mathrm{SiC}$ Hybrid Composite Using Agglomerative Hierarchical Clustering and KMeans. Silicon, 13, 961-972.

[12] Saxena P, Maheshwari A, Maheshwari S. Predictive modeling of brain tumor: A Deep learning approach. InInnovations in Computational Intelligence and
Computer Vision 2021 (pp. 275285). Springer, Singapore.

[13] Ezhilarasi, T. P., Dilip, G., Latchoumi, T. P., \& Balamurugan, K. (2020). UIP-A Smart Web Application to Manage Network Environments. In Proceedings of the Third International Conference on Computational Intelligence and Informatics (pp. 97-108). Springer, Singapore

[14] Tan L, Yu K, Bashir AK, Cheng X, Ming F, Zhao L, Zhou X. Toward real-time and efficient cardiovascular monitoring for COVID-19 patients by 5G-enabled wearable medical devices: a deep learning approach. Neural Computing and Applications. 2021 Jul 4: 1-4. 